\title{
Von W- und B-Fragen in der Versorgungsforschung
}

In der Medienwelt sind Nachrichten meist so aufgebaut, dass sie die sogenannten W-Fragen beantworten: «Was ist wann wo wem warum geschehen?» Davon lässt sich der Journalist in seiner Arbeit leiten. Auch in der Versorgungsforschung befassen wir uns mit W-Fragen: «Wie viele Ärzte haben wir?», «Was sind das für Ärzte, wo und was arbeiten sie?», und verwenden in der Suche nach Antworten die uns zur Verfügung stehenden Daten.

Wird heute über das Schweizer Gesundheitswesen diskutiert, kommt die Rede nach kurzer Zeit auf die ungenügende Datengrundlage und die dadurch erschwerte Planung. Egal, ob es um Managed Care, Anzahl Hausärzte, Angebot und Inanspruchnahme von Leistungen oder Qualität in der Schweiz geht, der Tenor lautet: Die Datengrundlage in der Schweiz ist mangelhaft und sollte verbessert werden. Erstellten Auswertungen folgt die Kritik, die Resultate seien irreführend. Der Versorgungsforscher versucht deshalb, die Datenbasis zu optimieren, verschiedene Datensätze zusammenzubringen, ihre Limitationen transparent darzustellen und vieles mehr, um die Qualität seiner Analysen zu erhöhen. Auch die FMH beteiligt sich an der Verbesserung von Datengrundlagen und an den vertieften Analysen bestehender und neuer Forschungsfelder. Sie hat ein Projekt gestartet, das die eigenen Daten über die Schweizer Ärzteschaft auf einen Stand bringen soll, der es ermöglicht, aktuelle und zukünftige Fragestellungen noch verlässlicher zu bearbeiten. Die FMH trägt auch zur weiteren Untersuchung der Versorgungssituation in der Schweiz bei, z.B., indem sie für nationale Projekte anerkannter Institutionen Daten über die Schweizer Ärzteschaft zur Verfügung stellt.
Aber Statistiken lösen die Probleme des Gesundheitssystems nicht einfach so. Als Beispiel dient die in den vergangenen Monaten in den Medien öfter zitierte Darstellung über die Zunahme der Ärzteschaft, wie sie in der Ärztestatistik ausgewiesen wird. Werden diese Zahlen veröffentlicht, folgt - neben der genannten Diskussion über die Qualität der Daten - sofort eine Auseinandersetzung über weitere Themen, wie z.B.:

- Sind die Kosten aufgrund der Zunahme an Ärztinnen und Ärzten gestiegen?

- Wird der Zulassungsstopp nicht seriös umgesetzt?

- Was leistet die heutige Anzahl Ärzte im Vergleich zur früheren Anzahl Ärzte (zeitlich und inhaltlich)?

Alle diese Themenkomplexe verdienen es, seriös untersucht zu werden. Aber damit ist es nicht getan. Die Antworten darauf schützen uns über kurz oder lang nicht, die dahinterstehenden Fragen anzugehen. Ein Beispiel: «Wie ist der Bedarf an Rheumatologen im Engadin?» Auf solche Fragen finden sich bisher kaum Antworten in den Statistiken, vielleicht, weil sich (wie in der Medienwelt) alle mit den W-Fragen beschäftigen? Und darüber hinaus vergessen oder verdrängen, dass in diesen Fragen ein anderes Wort vermehrt im Mittelpunkt stehen müsste, ein Wort mit B - B wie Bedarf? Die Versorgungsforschung kann hierzu einen Beitrag liefern, aber nicht allein: Die Partner im Gesundheitswesen, die Politik und letztendlich die Gesellschaft müssen sie gemeinsam finden, die Antworten auf die B-Fragen, die Fragen nach dem Bedarf.

Martina Hersperger, Leiterin Abteilung DDQ 